\title{
Spectroscopy of novel systems
}

From R.C. Thompson

\section{Atomic spectroscopy is alive and well thanks to the continuing development of novel experimental techniques and their application to new areas of interest.}

THOSE people who think that the subject of atomic spectroscopy was wrapped up several decades ago would have seen much evidence to the contrary at the recent EGAS conference held at Imperial College ${ }^{1}$. Completely new areas of spectroscopy are still arising, bringing with them new and interesting physics. For example, the study of Rydberg atoms (atoms where one electron is excited into a state of high principal quantum number) has blossomed recently, and an up-to-date review of this field was given by $\mathrm{C}$. Fabre (Ecole Normale Supérieure, Paris). Because many atomic properties scale as large powers of the effective principal quantum number $n^{*}$, Rydberg atoms can have large diameters (up to a fraction of a micrometre - large enough to be measured using a fine sieve), very long lifetimes and large polarizabilities. These facts make possible very unusual experiments - the detection of single microwave photons using Rydberg atoms as sensitive detectors; the modification of the spontaneous decay rate of atoms by putting them into a high-Q resonant microwave cavity; and even the possibility of constructing a single-atom laser (the usual many-body nature of laser action is made possible by the interaction of the atom with its images in the walls of the cavity). In experiments where a thermionic diode is used to detect ions formed by collisional ionization of laser excited Rydberg atoms, transitions to states with $n$ up to 200 have been studied.

A relatively new area of spectroscopy was reviewed by $G$. Soff (Gesellschaft für Schwerionen, Darmstadt). Super-heavy quasi atoms are created for very short periods $\left(10^{-20} s\right)$ in collisions between heavy projectile and target nuclei at energies of a few $\mathrm{MeV}$ per nucleon. As an example, at a total nuclear charge $Z=164$ (formed in $\mathrm{a} \mathrm{Pb}+\mathrm{Pb}$ collision), the nucleus created has a radius of $10 \mathrm{fm}$, while the radius of the $1 \mathrm{~s}$ electron wavefunction is $20 \mathrm{fm}$. Some unusual physical effects occur under such extreme conditions, such as the spontaneous emission of positrons during the collisions. This becomes energetically favourable at a combined charge $Z>173$, when the $1 \mathrm{~s}$ state enters the lower continuum of the Dirac Hamiltonian, that is, the binding energy becomes greater than the energy required for creation of an electron-positron pair. Measurements of

1. The Sixteenth European Group for Atomic Spectroscopy Conference was held at Imperial College, London on 10-13 July 1984. this and other effects in progress at GSI enable tests to be made of quantum electrodynamics in novel situations.

Another new development is the first observation of photon correlations in a true second-order two-photon decay (H.J. Duncan, University of Stirling). In this experiment, metastable hydrogen atoms (in the $2 \mathrm{~S}$ state) in an atomic beam decay emitting two photons in random directions. Events are recorded where one photon enters each of two detectors placed $180^{\circ}$ apart. Measurements of both the linear and circular polarization correlations between the two photons enable a test to be made of the celebrated Bell's inequality, which distinguishes the predictions of quantum mechanics from those of local realistic theories. As in other complementary experiments performed recently, these results favour quantum mechanics, but the controversy about whether or not quantum mechanics provides a complete picture still rages.

One of the highlights of the conference was the screening of a film showing the results of a series of calculations of the behaviour of an atom being irradiated by laser light (B.W. Shore, Imperial College London and Lawrence Livermore National Laboratory). The idealized two-level atom is a familiar concept, but here a slightly less idealized ten-level atom is treated, the results being displayed in the film as a two-dimensional plot of the density matrix of the atom as a function of

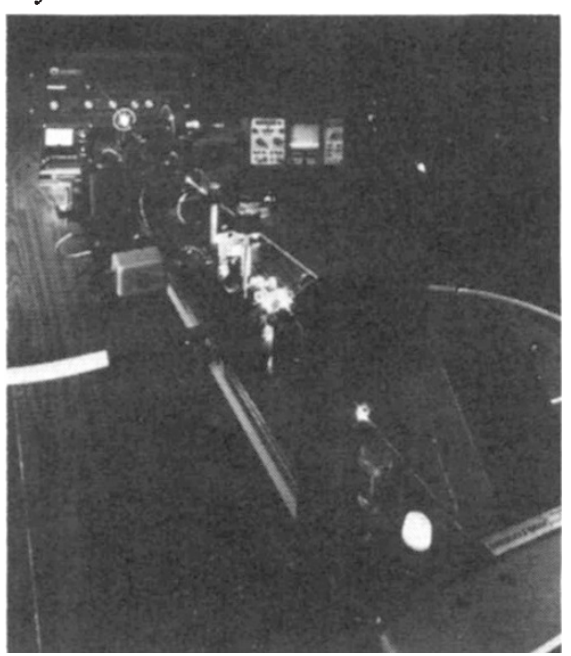

The Coherent 699-29 Autoscan, a self contained dye laser spectrometer ideally suited to highresolution absorption spectroscopy. The heart of the system is the Coherent 699-21 actively stabilized single-mode ring dye laser. Control is provided by an Apple II microcomputer, which can also be used for up to three channels of data acquisition. Circle no. 100 for further details. time. The phenomena illustrated include the periodic complete population inversion obtained with resonant coherent excitation and the gradual buildup of population in a specific excited level when the laser frequency corresponds to a multiphoton resonance. The film gave a valuable insight into the physical processes described by sets of equations treated usually only in an abstract mathematical manner.

Experiments using laser excitation were prominent at the conference and one striking example of the way the use of laser techniques is still growing is the application of coherent radiation (at wavelengths below $100 \mathrm{~nm}$ ) generated by four-wave mixing in $\mathrm{Kr}$ gas to the study of VUV transitions in $\mathrm{Xe}$, including the measurement of absolute wavelengths to 2 parts in $10^{6}$ ( T.J. Mcllrath, University of Maryland and National Bureau of Standards, Washington).

Most laser experiments, however, are performed in more conventional wavelength ranges. The availability of reliable single-mode tunable dye lasers covering the whole of the visible spectrum has meant that high resolution and power are now easily achieved in this region. Numerous Doppler-free laser techniques have been developed for the recording of spectral lines under many different source conditions; the laser has thus become an invaluable aid to the study of the structure of atoms and molecules.

A recent trend is for much atomic spectroscopy to be performed at large centralized facilities such as accelerators (used for example for the production of beams of atoms in various states of ionization) and nuclear reactors (for the creation of rare isotopes). This places more stringent requirements on the reliability of experimental apparatus, so that there has been a move in general towards computer controlled equipment and automated data acquisition. The use of computers also in the analysis of data has meant that more complex atomic systems can be investigated and this complements the theoretician's growing ability to perform calculations on such systems.

The EGAS conference demonstrated the broad range of the subject, with sessions covering topics as diverse as term analysis, isotope shifts, electron spectroscopy and collisional broadening.

R.C. Thompson is at the Division of Mechanical and Optical Metrology, National Physical Laboratory, Queens Road, Teddington, Middlesex TWII OLW, UK. 